\title{
Nova Scotia contemplates a continuum of coordinated, lifetime care for autism patients
}

Published at www.cmaj.ca on June 4

$\mathrm{T}$ he province of Nova Scotia is weighing the merits of establishing regional "total care" centres to treat the rising number of people diagnosed with autism spectrum disorder.

The centres would aggregate autism services under one roof, allowing sufferers and their families to easily access psychologists, occupational therapists, behaviour therapists, speech and language services, as well as a library of autism resources. Each centre would also house an "autism navigator," who would guide families through the treatment process.

There's a therapeutic benefit to providing such a continuum of care within one facility because many families struggle to find help following an autism diagnosis, concludes the province's Autism Management Advisory Team, which earlier this year released a report recommending 53 measures to help Nova Scotia better treat and support those living with the neurological disorder, which affects communication and social interaction.

"As a result, parents become confused, isolated, and have feelings of helplessness," stated the report, Lifespan Needs for Persons with Autism Spectrum Disorder, which was dedicated to James Delorey, an autistic boy who died in December 2009 after being lost for two days in the woods near his Cape Breton home.

The main idea, says the advisory team, is to offer "seamless" autism support from childhood through adulthood. "Although there is no known cure for autism, significant and lifechanging improvements can be achieved, if appropriate interventions and supports are put in place for the individual," stated the 129-page report (www.ednet.ns.ca/pdfdocs/autism_mgmt /Autism\%20Management\%20Advisory $\% 20$ Team\%20Report.pdf).

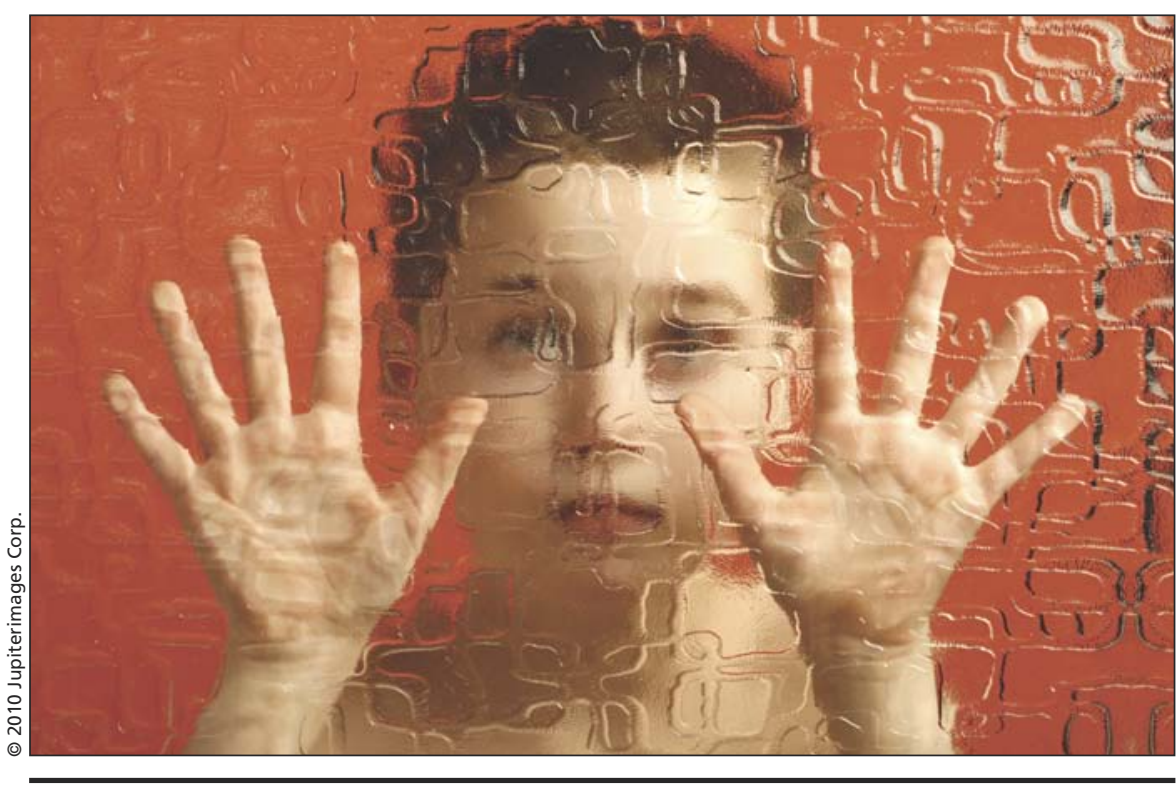

Autism Society Canada estimates that roughly 200000 Canadians are autistic.

"The unique and complex nature of autism compels us to think differently when we plan for support throughout the lifespan."

The government, though, won't deliver its initial response to the report until the fall.

But Susan Bryson, a psychologist and autism researcher in Halifax, NS, says the province should move down a "continuum of care" path that is slowly being adopted in Australia, the United Kingdom, the United States and parts of some provinces, such as British Columbia and Ontario.

Autism treatment typically involves numerous health care professionals in different locations and is not always available over the course of an autistic person's lifespan. "I can't emphasize enough how hard it is for families," says Bryson, who holds the Craig Chair in Autism Research at Dalhousie University and the Izaak Walton Killam Health Centre in Halifax. "The families have been telling us this for years: that it's best when services are coordinated. That way everyone wins."
The benefits quickly become evident when autism care is coordinated, says Dr. Rob Nicolson, a psychiatrist at the Child and Parent Resource Institute in London, Ontario, a facility which houses psychiatrists, a psychologist, behavioural therapists, social workers and speech and language therapists who collectively help treat as many as 500 autistic children and adolescents annually.

The facility, though, doesn't provide "lifespan" care - as recommended by the Nova Scotia report but only aids children and adolescents up to the age of 18 .

Still, the provincially funded centre saves families from being overwhelmed by the complexities of autism treatment, Nicholson says. "It's much easier to coordinate appointments and it's easier for the professionals to communicate with each other. Everyone is on the same page."

"If it can come to fruition in Nova Scotia, it will certainly push autism diagnosis and treatment ahead tremendously," he adds. 
Nova Scotia's advisory panel urged the creation of the centres because of soaring autism rates. Citing figures from the Centers for Disease Control and Prevention, they noted that the prevalence of autism is now 1 in 110, significantly higher than the 1 in 2500 rate found during the 1960s.

"This fact urges us to prepare for the increasing numbers of individuals diagnosed with autism," states the report, noting that approximately 9500 Nova Scotians have been diagnosed with an Autism Spectrum Disorder.

Catherine Rice, a behavioural scientist with the Centres for Disease Control and Prevention, says the rise in autism diagnoses cannot be fully explained. But she says a good portion of new autism diagnoses are the result of a broadened definition of the disorder.
Prior to the 1980s, only those exhibiting autism's classic symptoms (communication and behavioural difficulties and a lack of social interaction) were considered affected. Those symptoms remain the foundation of "classic" autism.

But the autism umbrella has since widened to include milder forms, says Dr. Marshalyn Yeargin-Allsopp, a medical epidemiologist at the Centers for Disease Control and Prevention. For example, it now includes Asperger syndrome, where the sufferer is socially impaired, but experiences typical language development.

Another difference between past and present autism diagnosis involves the presence of intellectual disabilities, adds Yeargin-Allsopp. During the 1960s and 1970s, the vast majority of those diagnosed with autism had an intellectual disability but today, only about $40 \%$ have one.

Rice says better diagnosis and detection also play a role in the current rise of cases.

Given those changes, is autism actually affecting more people now than it did decades ago?

Possibly, Rice says. "That's the crux of the question that we and others are trying to figure out. It has become clear that no single factor is explaining the increase. Unfortunately, we have a lot to learn about the causes of autism."

According to Autism Society Canada, roughly 200000 Canadians are living with an autism spectrum disorder. — Quentin Casey, Halifax, NS

DOI:10.1503/cmaj.109-3274 